\title{
1RXS J214303.7+065419/RBS 1774: A new Isolated Neutron Star candidate ${ }^{\star}$
}

\author{
L. Zampieri ${ }^{1,2}$, S. Campana ${ }^{3}$, R. Turolla ${ }^{2}$, M. Chieregato ${ }^{2}$ \\ R. Falomo ${ }^{1}$, D. Fugazza ${ }^{3}$, A. Moretti ${ }^{3}$, and A. Treves ${ }^{4}$
}

\footnotetext{
1 Osservatorio Astronomico di Padova, Vicolo dell'Osservatorio 5, 35122 Padova, Italy

2 Università di Padova, Dipartimento di Fisica, Via Marzolo 8, 35131 Padova, Italy

3 Osservatorio Astronomico di Brera, Via Bianchi 46, 23807 Merate, Italy

4 Università dell'Insubria, Dipartimento di Scienze, Via Valleggio 11, 22100 Como, Italy
}

Received 24 July 2001 / Accepted 14 August 2001

\begin{abstract}
We report on the identification of a new possible Isolated Neutron Star candidate in archival ROSAT observations. The source 1RXS J214303.7+065419, listed in the ROSAT Bright Source Catalogue as RBS 1774, is very soft, exhibits a thermal spectrum well fitted by a blackbody at $T \sim 90 \mathrm{eV}$ and has a low column density, $N_{\mathrm{H}} \sim 5 \times 10^{20} \mathrm{~cm}^{-2}$. Catalogue searches revealed no known sources in other energy bands close to the X-ray position of RBS 1774. Follow-up optical observations with NTT showed no peculiar object within the X-ray error circle. The absence of any plausible optical counterpart down to $m_{R} \sim 23$ results in an X-ray to optical flux ratio in excess of 1000 .
\end{abstract}

Key words. stars: individual: 1RXS J214303.7+065419/RBS 1774 - stars: neutron - X-rays: stars

\section{Introduction}

Over the last few years ROSAT observations led to the discovery of six very soft X-ray sources with quite peculiar characteristics. Among these are (i) blackbody-like spectrum with $T \sim 100 \mathrm{eV}$; (ii) exceedingly large X-ray to optical flux ratio, $f_{\mathrm{X}} / f_{V}>10^{3}$; (iii) low X-ray luminosity, $L_{\mathrm{X}} \approx 10^{30}-10^{31} \mathrm{erg} \mathrm{s}^{-1}$; (iv) low column density, $N_{\mathrm{H}} \sim 10^{20} \mathrm{~cm}^{-2}$; (v) no evidence for a binary companion; (vi) absence of large flux variations on time-scales from months to years (see e.g. Treves et al. 2000; Motch 2000 for reviews).

All these points, in particular the extreme values of $f_{\mathrm{X}} / f_{V}$ together with the small distances implied by the low column density, qualify these sources as potential, close-by Isolated Neutron Stars (INSs). Three sources have been found to pulsate with periods in the range 5-23 s, strengthening further the association with neutron stars (RX J1605.3+3249/RBS 1556, Haberl, private communication; RX J0720.4-3125, Haberl et al. 1997; RX J04020.0-5022, Haberl et al. 1999). Intensive follow-up campaigns led to the identification of an optical counterpart for RX J1856.5-3754 $\left(m_{V} \sim 25.6\right.$; Walter \& Matthews 1997; see also van Kerkwijk \& Kulkarni 2001) and, with less certainty,

Send offprint requests to: L. Zampieri

e-mail: zampieri@pd.astro.it

* Partially based on observations carried out at ESO, La Silla, Chile (67.H-0116(A)). for RX J0720.4-3125 $\left(m_{B} \approx 26\right.$; Motch \& Haberl 1998; Kulkarni \& van Kerkwijk 1998). Very recently, Walter (2001) obtained a parallax distance of $\sim 60 \mathrm{pc}$ for RX J1856.5-3754 with the Hubble Space Telescope, providing direct evidence that these sources should indeed be located within a few hundred parsecs.

Although it is now widely agreed that the six ROSAT sources are isolated neutron stars, their puzzling properties make the origin of their emission still uncertain. Up to now, two possibilities have been discussed: accretion of the interstellar medium onto old INSs or thermal emission from much younger cooling neutron stars. Due to the extreme similarity of the expected X-ray spectra, however, present data still do not allow an unambiguous determination of the nature of these sources (Treves et al. 2000). Statistical methods have been used to probe and compare the distributions of cooling and accreting INSs (Neühauser \& Trümper 1999; Popov et al. 2000), but, being severely hindered by the limited sample of detected sources, they fail to give a definite answer. The similarity in the periods has suggested a possible evolutionary link between Anomalous X-ray Pulsars, Soft Gamma-ray Repeaters and ROSAT INSs whereby the former may be the progenitors of the latter. If this is the case, RX J0720.4-3125 might be a magnetar kept hot by the dissipation of a superstrong magnetic field $\left(B \approx 10^{14}-10^{15} \mathrm{G}\right.$; Heyl \& Hernquist 1998; Heyl \& Kulkarni 1998).

The discovery of new INS candidates is of paramount importance to shed light on the properties of these 
sources and to constrain their distribution in the Galaxy. In this paper we report the identification of a new possible INS candidate in archival ROSAT observations. X-ray and optical follow-up observations are described in Sect. 2. Discussion on the nature of 1RXS J214303.7+065419/RBS 1774 and conclusions are reported in Sect. 3.

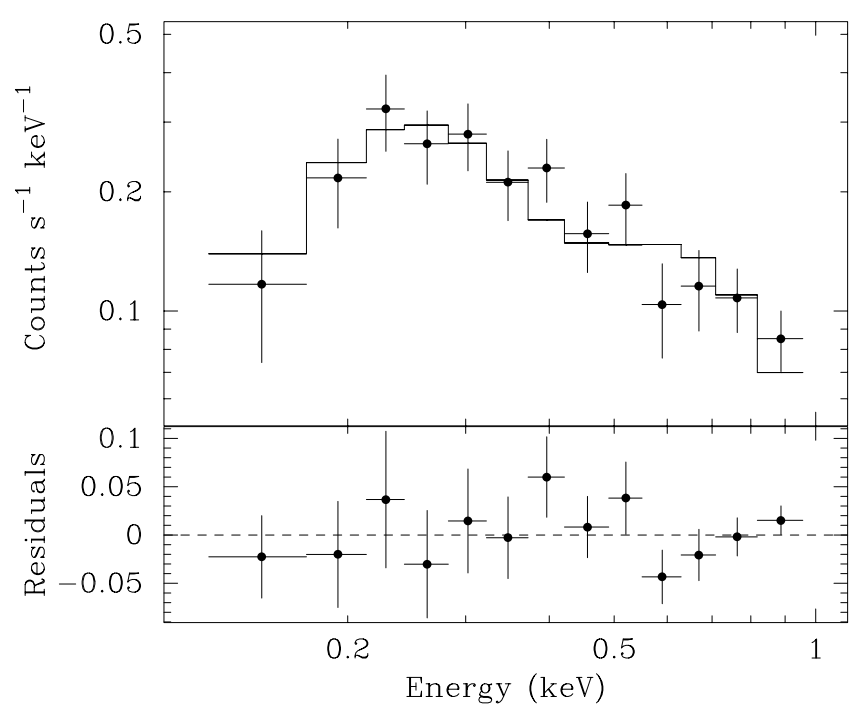

Fig. 1. The ROSAT PSPC spectrum of RBS 1774 with the best-fitting blackbody with $T=92 \mathrm{eV}$ and a low column density, $N_{\mathrm{H}}=4.6 \times 10^{20} \mathrm{~cm}^{-2}$.

\section{Observations}

\subsection{X-ray observations}

We re-examined archival ROSAT PSPC data looking for relatively bright sources with $f_{\mathrm{X}} / f_{V}>100$ and no likely counterpart on Digital Sky Survey fields. The search on the ROSAT Bright Survey (RBS; Fisher et al. 1998) catalogue yielded three sources with count rates $\gtrsim 0.1$ counts $^{-1}$ matching the adopted criteria. Two of them are the already known INS candidates RX J1605.3+3249/RBS 1556 (Motch et al. 1999) and 1RXS J130848.6+212708/RBS 1223 (Schwope et al. 1999). The remaining one, 1RXS J214303.7+065419/RBS 1774 (or 1WGA J2143.0+0655), was serendipitously detected by ROSAT in a PSPC pointing of the BL Lac object MSS 2143.4+0704 (Stocke et al. 1991). The 3.5 ks observation was performed in May 1991 (ROSAT pointing \# rp700045n00). RBS 1774 is located about $48^{\prime}$ off-axis, to the south-west of the position of MSS 2143.4+0704, and is present both in the WGA (White et al. 1994) and the ROSATSRC (Zimmermann 1994) catalogues. Examination of the NED and SIMBAD astronomical databases did not show any catalogued source within $5^{\prime}$ of the nominal RBS source position.

The analysis of the PSPC field provides a poor determination of the source position because of the large off-axis angle. The nominal positions in the WGA and
ROSATSRC catalogues differ by $\sim 30^{\prime \prime}$. In the short $(373 \mathrm{~s})$ exposure taken during the ROSAT All Sky Survey (RASS; Voges et al. 1996) the source is at a much smaller average off-axis angle so, albeit few photons were collected, it allows for a better position estimate. The RASS coordinates are again different from those of the PSPC field. For this reason we decided to run a simplified version of the Brera Multiscale Wavelet (BMW) algorithm (Lazzati et al. 1999; Campana et al. 1999) on the RASS field. The estimated position derived from our re-analysis of RASS data is $\mathrm{RA}=21^{\mathrm{h}} 43^{\mathrm{m}} 02.0^{\mathrm{s}}, \mathrm{DEC}=+06^{\circ} 54^{\prime} 26^{\prime \prime}(\mathrm{J} 2000)$, with a $90 \%$ uncertainty radius of $14^{\prime \prime}$.

Photons were extracted from a $6^{\prime} \times 3.5^{\prime}$ elliptical region centered on the source position in the pointed PSPC observation. A total of 763 photons were collected, providing $\sim 500$ net counts after background subtraction. The count rate is 0.18 counts $\mathrm{s}^{-1}$. The spectral analysis was carried out with XSPEC (v. 11.0.1). Energy channels were grouped into bins containing 50 photons each to have acceptable counting statistics. The background subtracted spectrum is very soft and we tried several single-component spectral models (see Fig. 1 and Table 1). All models provide a statistically acceptable fit to the data. However, the power-law index is unplausibly large, $\Gamma \sim 5.5$, and the Raymond-Smith model converges toward very low metal abundances $\left(Z<10^{-2} Z_{\odot}\right)$, a pure bremsstrahlung with a temperature too low for emission from intracluster gas. If the metal abundance is held fixed at solar, the fit is not acceptable $\left(\chi_{\text {red }}^{2}=4.7\right)$, ruling out emission from active stellar coronae. A fit with the synthetic spectra of Zampieri et al. (1995) for thermal emission from unmagnetized neutron star hydrogen atmospheres, accreting at low rates, was also attempted. Using this model, parameterized by the total luminosity in units of the Eddington luminosity (for a $1.4 M_{\odot}$ neutron star), a distance of $\sim 50 \mathrm{pc}$ is derived which is likely too small for the associated column density. The fit with a blackbody is acceptable and gives $T \sim 90 \mathrm{eV}, N_{\mathrm{H}} \sim 4.6 \times 10^{20} \mathrm{~cm}^{-2}$. These values are in reasonable agreement with what expected for thermal emission from an isolated neutron star. Furthermore, the value of the column density derived from the blackbody fit is the lowest among the fitted models and is well consistent with the total Galactic absorption in the source direction $\left(\sim 5 \times 10^{20} \mathrm{~cm}^{-2}\right.$, Dickey \& Lockman 1990). The $0.1-2.4 \mathrm{keV}$ unabsorbed flux is $8.7 \times 10^{-12} \mathrm{erg} \mathrm{s}^{-1} \mathrm{~cm}^{-2}$ (Table 1) and the total blackbody flux $9.1 \times 10^{-12} \mathrm{erg} \mathrm{s}^{-1} \mathrm{~cm}^{-2}$.

Despite the limited number of counts, a timing analysis has been attempted. After correcting the photon arrival times to the solar system baricenter, no statistically significant periodicity has been observed with a $3 \sigma$ upper limit on the pulsed fraction of $\sim 30 \%$ in the $1-100 \mathrm{~s}$ period range. A Kolmogorov-Smirnov test performed on the data set assigns a probability $<2 \sigma$ to the hypothesis that the source is variable. The source appears not to be variable also on longer time-scales ( months), as confirmed by the consistency of the count rate between the ROSAT All Sky Survey and the pointed PSPC observations. 
Table 1. X-ray spectral models.

\begin{tabular}{lcccc}
\hline Model & Parameters ${ }^{1}$ & $\begin{array}{c}N_{\mathrm{H}} \\
\left(10^{20} \mathrm{~cm}^{-2}\right)\end{array}$ & $\chi_{\text {red }}^{2}$ (d.o.f.) & $\begin{array}{c}\text { Flux }^{2}(0.1-2.4 \mathrm{keV}) \\
\left(\mathrm{erg} \mathrm{s}^{-1} \mathrm{~cm}^{-2}\right)\end{array}$ \\
\hline Blackbody & $k T=92_{-15}^{+19} \mathrm{eV}$ & $4.6_{-0.2}^{+0.2}$ & $0.83(10)$ & $8.7 \times 10^{-12}$ \\
Power law & $\Gamma=5.5_{-1.2}^{+1.2}$ & $11.3_{-0.3}^{+0.3}$ & $0.54(10)$ & $7.4 \times 10^{-10}$ \\
Raymond-Smith & $k T=168_{-41}^{+78} \mathrm{eV}, Z<10^{-2} Z_{\odot}$ & $7.3_{-0.2}^{+0.2}$ & $0.68(9)$ & $3.0 \times 10^{-11}$ \\
Zampieri et al. & $\log \left(L / L_{\mathrm{Edd}}\right)=-7.2_{-0.7}^{+0.8}$ & $6.9_{-0.1}^{+0.3}$ & $0.67(10)$ & $2.2 \times 10^{-11}$ \\
\hline
\end{tabular}

${ }^{1}$ Errors are at $90 \%$ confidence level.

${ }^{2}$ Fluxes are unabsorbed.

\subsection{Optical observations}

A deep image of the field containing RBS 1774 was taken on 27 May 2001 at the ESO $3.5 \mathrm{~m}$ New Technology Telescope (NTT) at La Silla. We used the ESO Multi Mode Instrument (EMMI) with a Tektronix CCD of $2048 \times 2048$ pixels yielding a field of view of $9.1^{\prime} \times 8.6^{\prime}$ at a resolution of $0.27^{\prime \prime} /$ pixel. The night was photometric with a seeing of about $0.8^{\prime \prime}$. Two exposures of $900 \mathrm{~s}$ each were obtained in the red $(R)$ bandpass. The data were reduced using standard ESO-MIDAS procedures for bias subtraction and flat-field correction. The two images were co-added and astrometry was performed using the USNO-A2 star catalogue which provides an absolute positional accuracy of $\sim 0.5^{\prime \prime}$ (Monet 1998). The central part of the field is shown in Fig. 2, where the $90 \%$ error circles relative to the different X-ray positions are also shown. As it can be seen, the error circles partially overlap within the error box provided by our re-analysis of RASS data. Although this is by no means conclusive, it lends support to our determination of the source position. Within our error circle two relatively bright objects (A and $\mathrm{B}$ in Fig. 2) are visible. The brighter one $(\mathrm{A})$ is the USNO-A2 star U0900_19784650, with catalogue magnitudes $m_{R}=15.5$ and $m_{B}=16.0$. The fainter object is not classified. The photometric analysis of these two objects in our NTT image was performed with the Sextractor program (Bertin \& Arnouts 1996). The photometric calibration was obtained from observations of Landolt standard stars (Landolt 1992). We derived the magnitudes of several stars in the field around the source position. The two objects within our error box have $m_{R}=15.40$ and $m_{R}=19.54$, respectively (see Table 2 ). Another faint object (D) is revealed inside the error circle at $m_{R}=22.77$. No other object is visible down to a limiting red magnitude of $\sim 25$, even if the bright star saturates a sizable fraction of the area.

Optical images of the field in the $B, V$ and $R$ filters were obtained on 29 June 2001 using the $2.5 \mathrm{~m}$ Nordic Optical Telescope (NOT) at La Palma. We used the Andalucia Faint Object Spectrograph and Camera (ALFOSC) with a Loral-Lesser CCD of $2048 \times 2048$ pixels yielding a field of view of $6.5^{\prime}$ at a resolution of $0.188^{\prime \prime} /$ pixel. The night was favorable to perform photometric observations, with a seeing of $0.8^{\prime \prime}$ (FWHM).
Standard data reduction was applied to the frames using IRAF. The photometric calibration was obtained from observations of Landolt standard stars (Landolt 1992). The $B$ and $V$ magnitudes of the field objects in Fig. 2 are given in Table 2. The color indices of the two objects within the BMW error box (A and B) are consistent with those of intermediate spectral type stars, whereas object $\mathrm{C}$ has a marginally negative $V-R$ and positive $B-V$. This behavior may be due to an unusual optical spectrum or to the presence of emission lines in the $V$ band.

Spectroscopic observations of objects A and B were obtained on 24 June 2001 at Loiano Observatory with BFOSC and a seeing of $1.8^{\prime \prime}$. We performed low-resolution (15 $\AA$, grism \#4) spectroscopy for a total exposure time of $3000 \mathrm{~s}$. After applying standard corrections, cosmic rays were removed and the sky-subtracted stellar spectra were obtained, corrected for atmospheric extinction. The signal to noise $(\mathrm{S} / \mathrm{N})$ ratio for the dim object was extremely low, providing an acceptable spectrum only above $4800 \AA$. No strong emission lines are observed, testifying that these objects are not Active Galactic Nuclei (see Fig. 3). Atmospheric absorption lines (A and B bands) are clearly visible in the spectra. Star A shows $\mathrm{H} \alpha$ and $\mathrm{H} \beta$ absorption lines, pointing to a moderately hot star (class $\mathrm{G}$ ). In the case of star B absorption around 5100-5200 $\AA$ is seen, possibly indicating a $\mathrm{K}$ main sequence star. These spectral classifications are in agreement with color indices derived from the photometry. The absence of $\mathrm{H} \alpha$ emission lines indicates that the two stars do not have active coronae.

\section{Discussion and conclusion}

The soft X-ray source 1RXS J214303.7+065419/RBS 1774 is an optically unidentified, relatively bright ROSAT source with peculiar properties. It is interesting to note that Cagnoni et al. (2001) have recently performed a systematic search for bright ROSAT sources with no optical counterpart on the Palomar Sky Survey from pointed PSPC observations (Blank Field Sources). However RBS 1774 does not appear in their list because it does not lie within the inner circle of the PSPC pointing adopted in their search. This source was probably included by Schwope et al. (1999) among the point-like RBS sources 


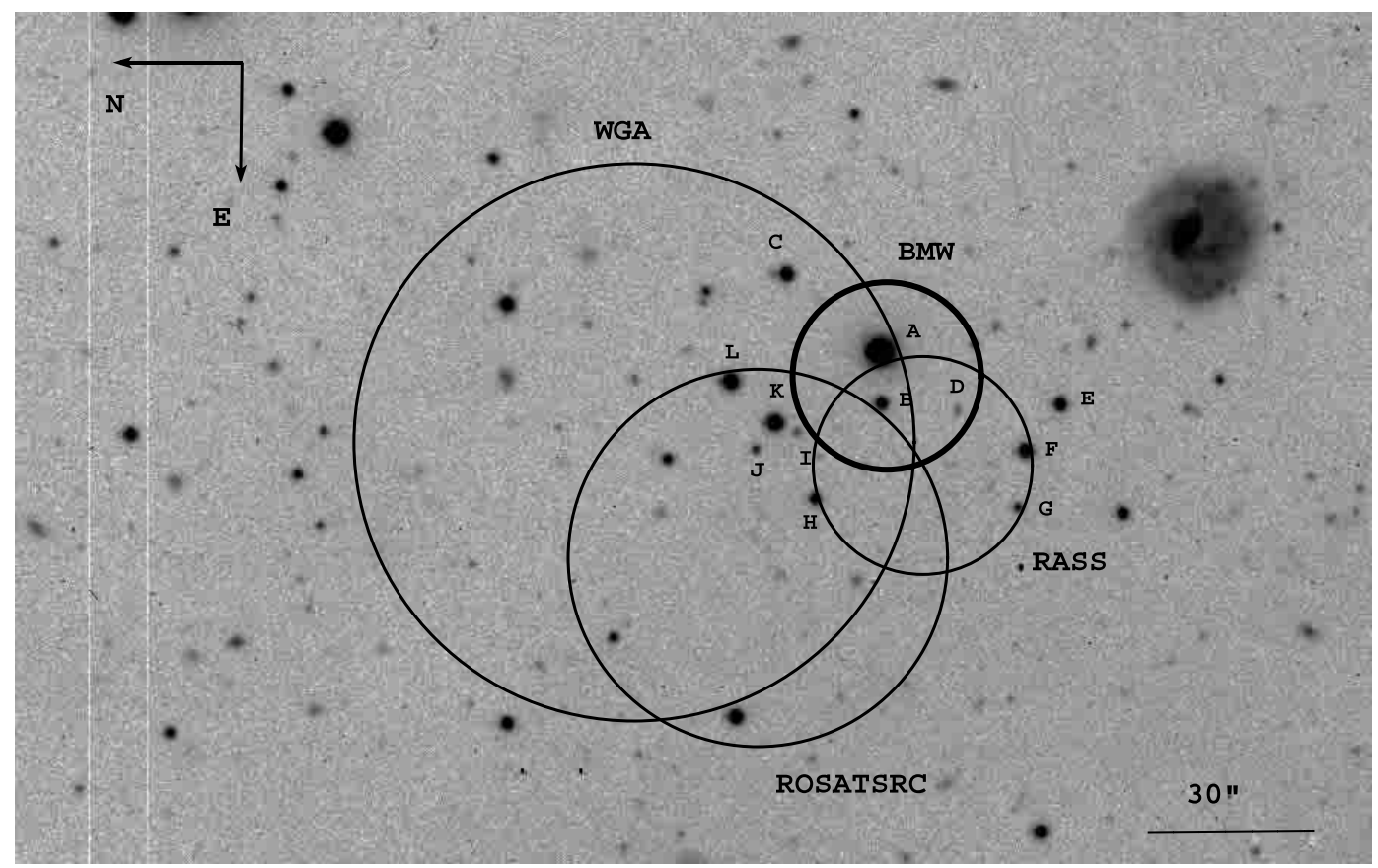

Fig. 2. The NTT $R$ field containing RBS 1774. The error circles refer to the different X-ray positions (see text).

with large $f_{\mathrm{X}} / f_{V}$ (the small triangle with the lowest hardness ratio in their Fig. 3 ).

As discussed in Sect. 2.1, the X-ray position of the source reported in the WGA and ROSATSRC catalogues is affected by significant uncertainties and is better determined from RASS data, that have been re-analyzed using a wavelet detection algorithm. The two objects contained within our estimated error box (A and B) have photometric and spectral properties consistent with those of low-main sequence stars, with no evidence for coronal activity. Therefore they are unlikely to be associated with the X-ray source. A third object $(\mathrm{C})$, which lies just outside the BMW $3 \sigma$ error box, has peculiar colors and might not be an ordinary star. Were it associated with the X-ray source, the inferred X-ray to optical flux ratio would be $\sim 100$, still consistent with emission from BL Lac objects, Low Mass X-ray Binaries and Cataclysmic Variables. However, the X-ray properties of RBS 1774 make these options unlikely. In fact, the very soft X-ray spectrum contrasts with emission from an extragalactic object or an X-ray binary, and the best-fit blackbody temperature and absence of variability argue against the identification with a Soft Polar. Most important, according to our wavelet detection analysis, the chance that object $\mathrm{C}$ is the counterpart of RBS 1774 is rather slim. No other plausible optical counterpart appears to be present within the error box, except possibly for the very faint object D. At present there are not sufficient information to establish if this object might actually be the counterpart of RBS 1774 . Taking as limiting magnitude for the optical counterpart that of field object $\mathrm{D}\left(m_{R} \sim 22.8\right)$ sets a lower limit to the X-ray to optical flux ratio of $\sim 1000$.
Table 2. Magnitudes of field objects in the $R, V$ and $B$ bands.

\begin{tabular}{cccccc}
\hline & $R$ & $V$ & $B$ & $V-R$ & $B-V$ \\
\hline A & $15.40 \pm 0.05$ & $15.95 \pm 0.05$ & $16.69 \pm 0.05$ & 0.6 & 0.7 \\
B & $19.54 \pm 0.01$ & $20.0 \pm 0.1$ & - & 0.5 & - \\
C & $19.00 \pm 0.01$ & $18.87 \pm 0.05$ & $19.0 \pm 0.1$ & -0.1 & 0.1 \\
D & $22.77 \pm 0.06$ & - & - & - & - \\
E & $19.38 \pm 0.01$ & $19.9 \pm 0.1$ & - & 0.5 & - \\
F & $19.05 \pm 0.01$ & $19.4 \pm 0.1$ & - & 0.4 & - \\
G & $21.32 \pm 0.03$ & $21.7 \pm 0.1$ & - & 0.4 & - \\
H & $20.10 \pm 0.01$ & $21.3 \pm 0.1$ & - & 1.2 & - \\
I & $22.05 \pm 0.05$ & - & - & - & - \\
J & $21.56 \pm 0.03$ & $22.2 \pm 0.1$ & - & 0.6 & - \\
K & $18.25 \pm 0.01$ & $18.96 \pm 0.05$ & - & 0.7 & - \\
L & $18.27 \pm 0.01$ & $19.03 \pm 0.05$ & $20.0 \pm 0.1$ & 0.8 & 1.0 \\
\hline
\end{tabular}

Star A saturates the NTT $R$ image and we adopt the NOT value. Estimated errors of $0.05-0.1 \mathrm{mag}$ in the NOT values are due to systematic errors and uncertainties in the standard calibration.

The very large inferred $f_{\mathrm{X}} / f_{V}$ of RBS 1774 rules out any known class of X-ray sources but Isolated Neutron Stars. In fact, the X-ray properties are remarkably similar to those of other INS candidates detected so far by ROSAT. The soft spectrum $(T \sim 90 \mathrm{eV})$, low column density $\left(N_{\mathrm{H}} \sim 5 \times 10^{20} \mathrm{~cm}^{-2}\right)$ and absence of variability in the X-ray data are consistent with this interpretation. Neglecting intrinsic absorption and considering a standard value for the average density of the interstellar medium in the source direction $\left(n_{\mathrm{e}} \sim 0.6 \mathrm{~cm}^{-3}\right)$, the inferred column density allows us to derive a rough estimate for the 


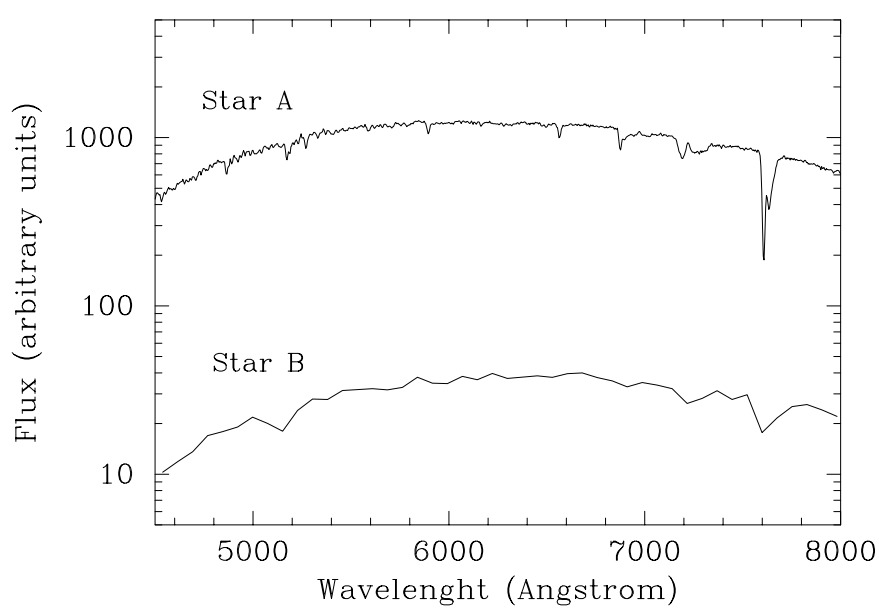

Fig. 3. The Loiano spectrum of stars A and B. Flux is in arbitrary units. Strong absorption lines are due to atmospheric absorption.

source distance of about $300 \mathrm{pc}$. Given that $N_{\mathrm{H}}$ is close to the total Galactic contribution along the line of sight, this distance should be considered a lower limit. The source luminosity is $L \sim 10^{32}(\mathrm{~d} / 300 \mathrm{pc})^{2}$ erg s ${ }^{-1}$, consistent with both a young, cooling and a low velocity, accreting neutron star. If the source is an old, accreting neutron star, the accretion flow may be in part channelled to the magnetic poles. The absence of modulation in the X-ray flux at the star spin frequency in the ROSAT data may simply be due to the low counting statistics and does not rule out this possibility. In fact, our derived upper limit to the pulsation amplitude $(\sim 30 \%)$ is by no means conclusive and a modulation may well be present with a lower rms amplitude.

The inferred bolometric flux and best fit blackbody temperature make it possible to estimate the radius of the neutron star as a function of distance. Neglecting gravitational redshift, it is: $R=3.2 f^{-1 / 2} \gamma^{2}(d / 300 \mathrm{pc}) \mathrm{km}$. Here $f$ is the fraction of the emitting area and $\gamma$ the intrinsic X-ray spectral hardening factor. The derived upper limit to the pulsation amplitude implies $f \sim 1$. The actual value of $\gamma$ depends on radiative transfer effects across the neutron star atmosphere and is significantly affected by the chemical composition and, possibly, the star magnetic field. Given that $\gamma \geq 1$, by using a realistic value for the neutron star radius it is possible to derive an upper limit to the source distance of $\sim 900(R / 10 \mathrm{~km})$ pc. Higher quality X-ray spectra will allow us to better constraint the spectral distribution and hence the value of $\gamma$.

In addition to deeper optical observations, an accurate re-determination of the X-ray position and spectrum, and a systematic search for periodicities are definitely required to improve our understanding of RBS 1774. In particular, the unprecedented positional accuracy and spectral resolution of the instruments on board the Chandra and XMM satellites provide a unique opportunity to confirm the identification of RBS 1774 with the seventh known Isolated Neutron Star.
Acknowledgements. We thank G. L. Israel for providing us with the software to compute the limit on the pulsed fraction, E. Bertone for his help in reducing spectral data, M. Cropper for a helpful discussion on the properties of Soft Polars and the referee Marten van Kerkwijk for useful comments. Part of the optical data presented here have been taken using ALFOSC, which is owned by the Instituto de Astrofisica de Andalucia (IAA) and operated at the Nordic Optical Telescope under agreement between IAA and the NBIfAFG of the Astronomical Observatory of Copenhagen. Work partially supported by the Italian Ministry for Education, University and Research (MIUR) under grant COFIN-2000-MM02C71842.

\section{References}

Bertin, E., \& Arnouts, S. 1996, A\&AS, 117, 393

Bregoli, G., Federici, L., Merighi, R., et al. 1987, in ESOOHP Workshop on the Optimization of the Use of CCD Detectors in Astronomy Proc. A88-13301 03-89 (Garching: ESO), 177

Cagnoni, I., Celotti, A., Elvis, M., Kim, D.-W., \& Nicastro, F. 2001, in Proc. of the Fourth Italian Conference on AGNs (Mem. SAIt), in press [astro-ph/0006257]

Campana, S., Lazzati, D., Panzera, M. R., \& Tagliaferri, G. 1999, ApJ, 524, 423

Dickey, J., \& Lockman, F. 1990, ARA\&A, 28, 215

Fischer, J.-U., Hasinger, G., Schwope, A. D., et al. 1998, AN, 319,347

Haberl, F., Motch, C., Buckley, D. A. H., Zickgraf, F.-J., \& Pietsch, W. 1997, A\&A, 326, 662

Haberl, F., Pietsch, W., \& Motch, C. 1999, A\&A, 351, L53

Heyl, J. S., \& Hernquist, L. 1998, MNRAS, 297, L69

Heyl, J. S., \& Kulkarni, S. R. 1998, ApJ, 506, L61

Kulkarni, S. R., \& van Kerkwijk, M. H. 1998, ApJ, 507, L49

Landolt, A. U. 1992, AJ, 104, 340

Lazzati, D., Campana, S., Rosati, P., Panzera, M. R., \& Tagliaferri, G. 1999, ApJ, 524, 414

Monet, D. G. 1998, AAS Meeting \#193, \#120.03

Motch, C., Haberl, F., Zickgraf, F.-J., Hasinger, G., \& Schwope, A. D. 1999, A\&A, 351, 177

Motch, C. 2000, in Bologna X-ray Astronomy 1999, in press [astro-ph/0008485]

Motch, C., \& Haberl, F. 1998, A\&A, 333, L59

Neühauser, R., \& Trümper, J. E. 1999, A\&A, 343, 151

Popov, S. B., Colpi, M., Prokhorov, M. E., Treves, A., \& Turolla, R. 2000, ApJ, 544, L53

Schwope, A. D., Hasinger, G., Schwarz, R., Haberl, F., \& Schmidt, M. 1999, A\&A, 341, L51

Stetson, P. B. 1987, PASP, 99, 191

Stocke, J. T., Morris, S. L., Gioia, I. M., et al. 1991, ApJS, 76, 813

Treves, A., Turolla, R., Zane, S., \& Colpi, M. 2000, PASP, 112, 297

van Kerkwijk, M. H., \& Kulkarni, S. R. 2001 A\&A, in press [astro-ph/0106265]

Voges, W., Aschenbach, B., Boller, T., et al. 1996, IAU Circ., 6420

Walter, F. M. 2001, ApJ, 549, 433

Walter, F. M., \& Matthews, L. D. 1997, Nature, 389, 358

White, N. E., Giommi, P., \& Angelini, L. 1994, IAU Circ., 6100 http://wgacat.gsfc.nasa.gov

Zampieri, L., Turolla, R., Zane, S., \& Treves, A. 1995, ApJ, 439,849

Zimmermann, H.-U. 1994, IAU Circ., 6102 